\title{
MorphoCol: A Powerful Tool for the Clinical Profiling of Pathogenic Bacteria
}

\author{
Ana Margarida Sousa, Anália Lourenço, and Maria Olívia Pereira*
}

\begin{abstract}
Pathogenicity, virulence and resistance of infection-causing bacteria are noteworthy problems in clinical settings, even after disinfection practices and antibiotic courses. Although it is common knowledge that these traits are associated to phenotypic and genetic variations, recent studies indicate that colony morphology variations are a sign of increased bacterial resistance to antimicrobial agents (i.e. antibiotics and disinfectants) and altered virulence and persistence.

The ability to search for and compare similar phenotypic appearances within and across species is believed to have vast potential in medical diagnose and clinical decision making. Therefore, we are developing a novel phenotypic ontology, the Colony Morphology Ontology (CMO), to share knowledge on the colony morphology variations of infection-causing bacteria. A study on the morphological variations of Pseudomonas aeruginosa and Staphylococcus aureus strains, two pathogenic bacteria associated with nosocomial infections, supported the development of CMO. We are also developing a new Web-based framework for the modelling and analysis of biofilm phenotypic signatures, supported by the CMO. This framework, named MorphoCol, will enable data integration and interoperability across research groups and other biological databases.
\end{abstract}

Keywords: infection-causing bacteria, biofilms, colony morphology, phenotype ontology, microbial database.

\section{Introduction}

In the clinical context, bacteria can be isolated from various sources such as patients, indwelling medical devices (e.g. catheters, prostheses, pacemakers,

Ana Margarida Sousa · Anália Lourenço · Maria Olívia Pereira

IBB - Institute for Biotechnology and Bioengineering,

Centre of Biological Engineering, University of Minho,

Campus de Gualtar, 4710-057 Braga, Portugal

e-mail: \{anamargaridasousa, analia, mopereira\} adeb.uminho.pt

* Corresponding author.

M.P. Rocha et al. (Eds.): 6th International Conference on PACBB, AISC 154, pp. 181-188. springerlink.com

(C) Springer-Verlag Berlin Heidelberg 2012 
endotracheal tubes), biological fluids (e.g. blood, urine and sputum) and abiotic surfaces (e.g. clinical and medical equipment and air). In addition, bacteria are usually isolated from biofilms, i.e. organised microbial communities entrapped on protective polymeric matrices, which are formed in a variety of biotic [1] and abiotic surfaces [2].

Bacterial persistence due to biofilm formation is a noteworthy downside in clinical settings, challenging disinfection and antibiotic procedures. Recent studies have pointed out colony morphology variations as a sign of increased resistance to antimicrobial agents (i.e. antibiotics and disinfectants) as well as of altered virulence traits and persistence of infection-causing microorganisms. Small colony variants (SCV) [3-5] and mucoid variants [6, 7] are examples of this phenomenon - e.g. the reduced size and the mucoidity of colony variants appear to be linked to a higher resistance to some significant antibiotics of medical use, such as vancomicin, kanamycin and ciprofloxacin.

Colony observation has thus become a crucial asset to medical diagnose and clinical decision making. However, the characterization has to go well beyond the simple identification of the microorganisms involved and some qualitative or quantitative description of the altered colony morphology. Despite of the simplicity of colony observation procedures, they often diverge on the experimental settings (namely, the conditions of cultivation) and colony morphology is likely to be altered under different conditions $[8,9]$.

The development of computational tools in assistance of phenotypic data deposition and further comparison is the key to integrate phenotypic observations into clinical routines. The challenge lays on establishing standard laboratorial guidelines and consensual criteria to identify the morphological characteristics of the colonies and then, creating a suitable and unambiguous computational representation for both the experiment profile and microscopic observations.

This work proposes the Colony Morphology Ontology (CMO), a novel ontology in support of the comprehensive annotation of colony phenotypic studies. This ontology supports MorphoCol, a Web-based framework suitable for the modelling and analysis of biofilm phenotypic signatures. The framework is being submitted to in-house testing and will be soon made publicly available at http://stardust.deb.uminho.pt/morphocol/.

\section{The Colony Morphology Ontology}

One of the signs of bacterial adaptability to aggressive environments is the change between distinct phenotypes similar to a mechanism On/Off. This changeability is augmented when bacteria face antimicrobial pressures or switch their mode of growth to the biofilm state [10]. A rapid tool to notice this variability is the spread of bacterial cultures on solid media and the observation of the morphology of the colonies adopted by bacteria. But, in order to take full advantage of these outputs, researchers are in need of controlled vocabulary to annotate the experimental settings and the parameters used to characterise the morphotypes unequivocally. 
The rationale of the CMO is to unify the vocabulary commonly used to describe colony morphologies within and between research and clinician groups and therefore being able to perform large-scale study comparison. This ontology is a module of a more comprehensive ontology on biofilm studies that describes the overall concept of biofilm signature as being a set of information on the microorganisms, the laboratory procedures, the antimicrobial susceptibility and virulence profiles (Fig. 1 - the top frame).

The hierarchical structure of the ontology is represented by a directed acyclic graph and the terms hold relationships of the type "part_of" - which represent part-whole relationships - and the type "is_a" - defining subtypes. The "microorganism" sub-tree (Fig. 1-a) embraces the identification of the infection-causing microorganism regarding the reference collection and the source from which it has been isolated (patient, indwelling devices, medical surfaces, etc.). Microbiologists need a bacteria identification to be able to compare studies and extrapolate the main phenotypic conclusions, i.e. it is not reasonable to consider the comparisons between different bacterial species.

The "experimental procedure" sub-tree (Fig. 1-b) encompasses the subclasses "growth assay", "plating assay" and "stress assay", "molecular assay", "omics assay" and "in vivo assay". A growth assay characterises a set of parameters associated to bacterial growth, such as growth media, temperature, $\mathrm{pH}$, time of growth, aeration, cell mode of growth, etc. Stress assays study the antimicrobial action (biocide, bacteriostatic and surfactant) of chemical and physical stressors on biofilms. Plating assay include the operational parameters associated to the spread of the bacterial cultures onto solid media and the development of the colonies. Molecular, 'omics' and in vivo assays address the use of high-throughput methods, such as polymerase chain reaction (PCR), expressing cloning, DNA arrays, MALDI-MS, gel electrophoresis, and in vivo techniques and conditions that may be used to confirm the preliminary identification and performs the comprehensive characterisation of the morphotypes. Again, results are comparable if and only if they were produced under the same culture and plating conditions.

The CMO sub-tree contains one root term "colony morphology" with two direct descendants: the terms "qualitative measure" and "quantitative measure" (Fig. 1-c). Colony morphology is firstly analysed in terms of qualitative parameters such as form (circular, irregular, filamentous, etc.), margin (entire, undulated, curled, etc.), surface (homogeneous, heterogeneous), texture (smooth, rough), elevation, size and colour. Then, it is quantified in terms of frequency, i.e. the number of colonies of a given morphotype variant per the total number of colonies in the plate.

The "antimicrobial susceptibility" sub-tree reports the identification of the morphotype according its tolerance to a range (and concentrations) of antimicrobials (antibiotics, disinfectants and biocides). The "virulence" sub-tree profiles the pathogenicity of colony variants in terms of virulence factors such as motility, enzymes production, tissue invasion ability and so on. 


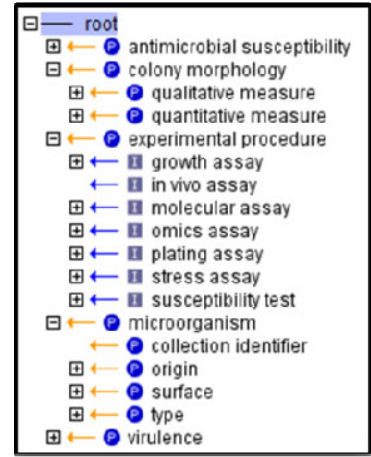

a)

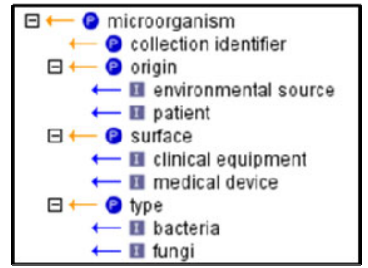

b)

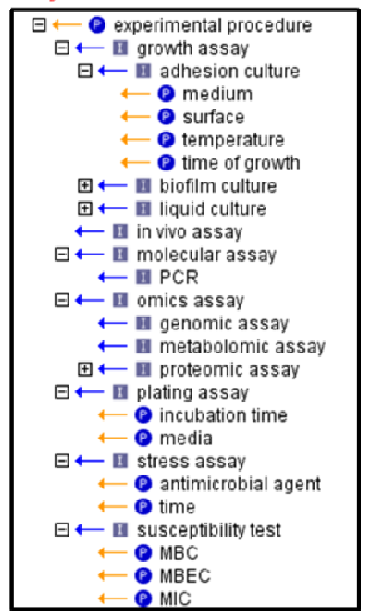

c)

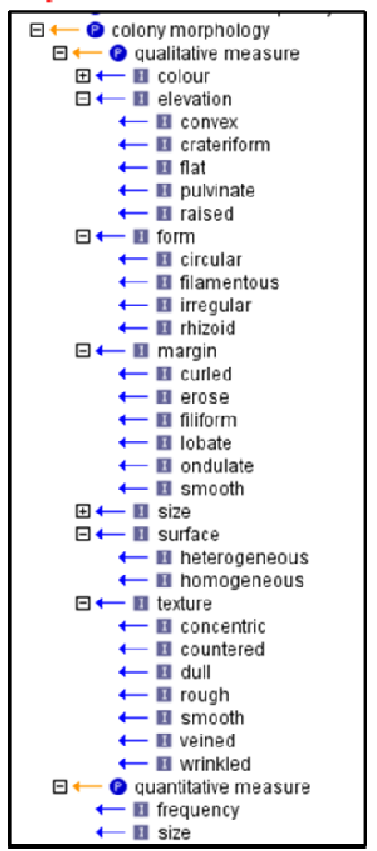

Fig. 1 The hierarchical structure of the ontology on biofilm studies. A profile on the morphology of the colony encompasses information on the microorganisms (a), the experimental assays performed (b) and the morphological characterisation (c).

\section{Results and Discussion}

The demonstration of the CMO and the development of the modelling and analysis framework are supported by an in-house study on the morphological variations of three pathogenic strains - P. aeruginosa ATCC 10145, a clinical isolated from 
medical equipment (PAI), and S. aureus ATCC 25923. The aim of this study is to evaluate the impact of experimental settings (in particular the solid media growth and incubation time) and the susceptibility profile of the colonies.

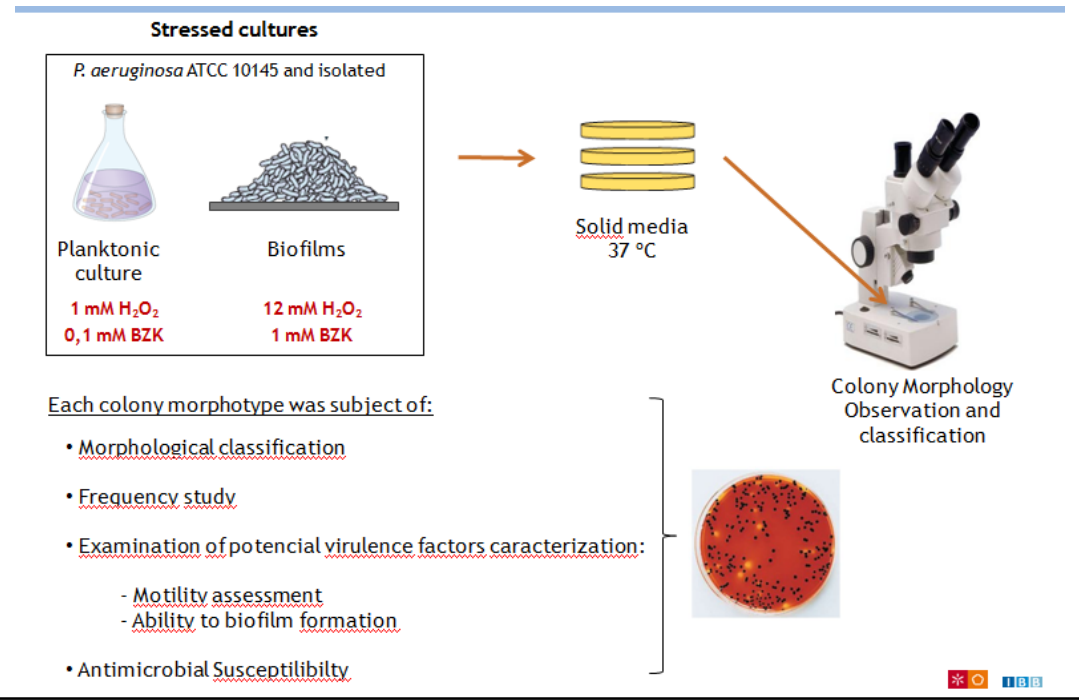

To include in CMO annotation: persistence of $P$. aeruginosa biofilms after hydrogen peroxide stress; colony diversity of $S$. aureus on different solid growth media; antibiotic susceptibility of different types of SCV.

Fig. 2 The experimental scheme to obtain, identify and characterize colonies of unstressed and stressed cultures. Data obtained on virulence and antimicrobial resistance about each morphotype was attached to experiment signature.

As illustrated in Fig. 2, bacteria are cultured on tryptic soy broth (TSB) and agar (TSA) media at $37^{\circ} \mathrm{C}$. During a period of 24 hours, bacteria are cultured in suspension (planktonic state) and in biofilm. Next, planktonic and biofilm cells are exposed to different antimicrobial agents of clinical use, namely to peroxide hydrogen and benzalkonium chloride. Then, non-stressed and stressed planktonic and biofilm cells are spread onto different solid media to observe and characterise the colonies at different incubation times. The observation of colony morphology was supported by directly placing petri plates on magnifying glass and photographed.

For our use case, it was possible to observe that the morphology of the colonies was strongly dependent of the experimental conditions. Data obtained revealed that the morphology of colonies was strongly dependent of the strain, cell mode of growth, type of solid media and incubation time. For only three bacterial strains it was observed more than one hundred distinct colonies. Also, the stressed cultures of all the strains have exhibited colony diversity. Fig. 3 presents some examples of the colonies observed. 


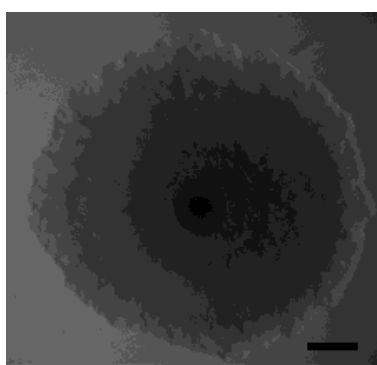

Strain: P. aeruginosa ATCC Diameter: $1,5 \mathrm{~cm}$ Colour: yellow Colony classification: LIRC

(a)

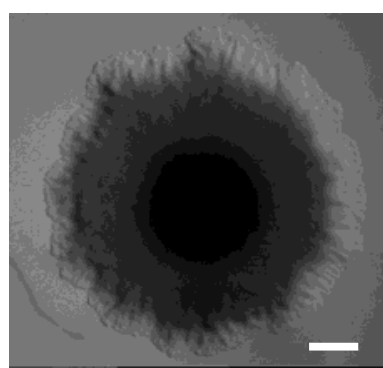

Strain: Isolated $P$. aeruginosa Diameter: $0,2 \mathrm{~cm}$

Colour: light brown Colony classification: RSCV

(d)

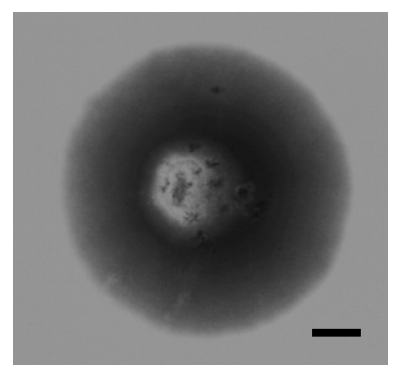

Strain: Isolated $P$. aeruginosa Diameter: $1,2 \mathrm{~cm}$

Colour: light brown

Colony classification: LRSC

(b)

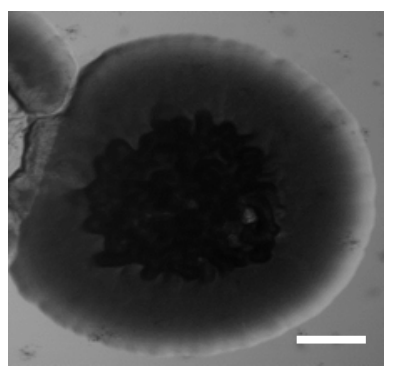

Strain: Isolated P. aeruginosa

Diameter: $0,2 \mathrm{~cm}$

Colour: light brown

Colony classification: $\mathrm{SwSCV}$

(e)

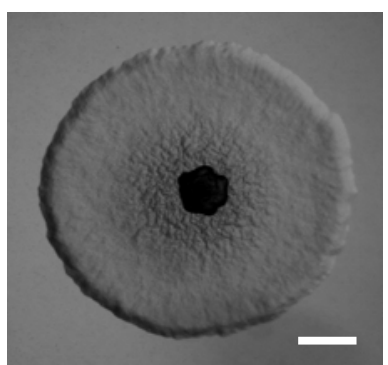

Strain: Isolated $P$. aeruginosa Diameter: $0,2 \mathrm{~cm}$ Colour: light brown Colony classification: RSCV

(c)

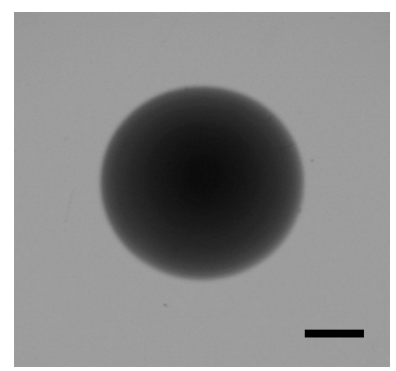

Strain: S. aureus ATCC

Diameter: $0,25 \mathrm{~cm}$

Colour: light brown

Colony classification: SCV

(f)

Fig. 3 Examples of colony morphology diversity and the corresponding CMO annotations: (a) larger (more than $3 \mathrm{~mm}$ of diameter), irregular and rough colonies (LIRC) formed by $P$. aeruginosa ATCC; (b) larger, regular and smooth colonies (LRSC) with craters formed by the isolated P. aeruginosa; (c), (d) and (e) are SCV (less than $3 \mathrm{~mm}$ of diameter) formed by isolated $P$. aeruginosa under antimicrobial stress and (f) formed by $S$. aureus ATCC. The SCV exhibited distinct surfaces: (c) and (d) present a rough surface (RSCV), (e) has a smooth and wrinkled surface (SwSCV) and (f) has smooth surface (SSCV). The coloured bar indicates the scale of the image: black bars $=1 \mathrm{~mm}$, white bars $=0,5 \mathrm{~mm}$.

These data provide novel insights into the proteomic alterations that occur during the complex process of morphotype switching, and lend support to the idea that this is associated with a fitness advantage in vivo. 
Table 1 The ciprofloxacin susceptibility profile of the SCV of $P$. aeruginosa ATCC and isolated $P$. aeruginosa. Analytical assessment: minimal inhibitory concentration (MIC) and minimal bactericidal concentration (MBC).

\begin{tabular}{cccc}
\hline \multirow{2}{*}{ Microorganism } & Morphotype & \multicolumn{2}{c}{ Ciprofloxacin $(\mathbf{m g} / \mathrm{L})$} \\
\cline { 3 - 4 } & & & \\
\hline P. aeruginosa ATCC & MBC \\
\cline { 3 - 4 } Isolated P. aeruginosa & LIRC & 0,1563 & 0,625 \\
& & & \\
& LISC & 0,0391 & 0,625 \\
& SrSCV & 0,0781 & 1,25 \\
& RSCV & 0,0781 & 0,625 \\
& SwSCV & 0,0781 & 1,25 \\
\hline
\end{tabular}

\section{Conclusions and Future Work}

Rapid identification of pathogenic microorganisms is crucial in medical diagnosis and clinical decision-making. Specifically, the ability to search for and compare similar phenotypic appearances within and across species is believed to have vast clinical potential. In this work, we propose a novel ontology on colony morphology - the CMO - as a step to the development of a fully integrated modelling and analysis framework for biofilm signatures. This approach has the potential to facilitate to efforts to link details on colony morphologies to specific environmental stressors and make our understanding of adaptive evolution more comprehensive.

Further development and refinement of the CMO will occur continuously and in parallel with phenotype annotation; thus, the evolution of the ontology will reflect the complexity with which phenotypes are described in biofilm research. The creation of terms will be based on need, specifically by curation of primary literature, by annotation of in-house phenotypic studies and by expert input from members of the biofilm research community.

Based on CMO, a public platform - MorphoCol - is under construction for deposition, description and characterization of colony morphologies. Thus, MorphoCol will help the consistent representation of colony morphology data.

Acknowledgements. The financial support from IBB-CEB and Fundação para a Ciência e Tecnologia (FCT) and European Community fund FEDER, through Program COMPETE, in the ambit of the FCT project "PTDC/SAU-SAP/113196/2009/ FCOMP-01-0124FEDER-016012" and Ana Margarida Sousa PhD Grant (SFRH/BD/72551/2010) are gratefully acknowledged. 


\section{References}

1. Burmolle, M., Thomsen, T.R., Fazli, M., Dige, I., Christensen, L., Homoe, P., Tvede, M., Nyvad, B., Tolker-Nielsen, T., Givskov, M., Moser, C., Kirketerp-Moller, K., Johansen, H.K., Hoiby, N., Jensen, P.O., Sorensen, S.J., Bjarnsholt, T.: Biofilms in chronic infections - a matter of opportunity - monospecies biofilms in multispecies infections. FEMS Immunol. Med. Microbiol. 59(3), 324-336 (2010)

2. Donlan, R.: Biofilms and device-associated infections. Emerging Infectious Diseases 7(2), 277-281 (2001)

3. Kirisits, M.J., Prost, L., Starkey, M., Parsek, M.: Characterization of colony morphology variants isolated from Pseudomonas aeruginosa biofilms. Appl. Environ. Microbiol. 71(8), 4809-4821 (2005)

4. Proctor, R.A., Peters, G.: Small colony variants in staphylococcal infections: diagnostic and therapeutic implications. Clin. Infect. Dis. 27(3), 419-422 (1998)

5. Haussler, S., Tummler, B., Weissbrodt, H., Rohde, M., Steinmetz, I.: Small-colony variants of Pseudomonas aeruginosa in cystic fibrosis. Clin. Infect. Dis. 29(3), 621-625 (1999)

6. Hogardt, M., Heesemann, J.: Adaptation of Pseudomonas aeruginosa during persistence in the cystic fibrosis lung. Int. J. Med. Microbiol. 300(8), 557-562 (2010)

7. Lyczak, J.B., Cannon, C.L., Pier, G.B.: Lung infections associated with cystic fibrosis. Clin. Micro-Biol. Rev. 15(2), 194-222 (2002)

8. Sousa, A.M., Loureiro, J., Machado, I., Pereira, M.O.: The role of antimicrobial stress on Pseudo-monas aeruginosa colony morphology diversity, tolerance and virulence. In: International Conference on Antimicrobial Research, Valladolid, Spain (2010)

9. Sousa, A.M., Loureiro, J., Machado, I., Pereira, M.: In vitro adaptation of P. aeruginosa: colony morphology variants selection and virulence characterization. In: International Conference on BIOFILMS IV, Winchester, England (2010)

10. Sousa, A.M., Machado, I., Pereira, M.O.: Phenotypic switching: an opportunity to bacteria thrive. In: Mendez-Vilas, A. (ed.) Science Against Microbial Pathogens: Communicating Current Research and Technological Advances (2011) (accepted)

11. Starkey, M., Hickman, J.H., Ma, L., Zhang, N., De Long, S., Hinz, A., Palacios, S., Manoil, C., Kirisits, M.J., Starner, T.D., Wozniak, D.J., Harwood, C.S., Parsek, M.R.: Pseudomonas aeruginosa rugose small-colony variants have adaptations that likely promote persistence in the cystic fibrosis lung. J. Bacteriol. 191(11), 3492-3503 (2009)

12. Seaman, P.F., Ochs, D., Day, M.J.: Small-colony variants: a novel mechanism for triclosan resistance in methicillin-resistant Staphylococcus aureus. J. Antimicrob. Chemother. 59(1), 43-50 (2007) 